Stud. Univ. Babeş-Bolyai Math. 66(2021), No. 1, 5-15

DOI: 10.24193/subbmath.2021.1.01

\title{
Applications of implicit parametrizations
}

\author{
Dan Tiba
}

Dedicated to Professor Gheorghe Moroşanu on the occasion of his 70th anniversary.

\begin{abstract}
We review several applications of the implicit parametrization theorem in optimization. In nonlinear programming, we discuss both new forms, with less multipliers, of the known optimality conditions, and new algorithms of global type. For optimal control problems, we analyze the case of mixed equality constraints and indicate an algorithm, while in shape optimization problems the emphasis is on the new penalization approach.
\end{abstract}

Mathematics Subject Classification (2010): 34A34, 49K21, 49Q10, 26 B10.

Keywords: Implicit functions and implicit parametrizations, optimization, optimal control, shape optimization.

\section{Introduction}

In several papers $[17,10,19]$, a constructive extension was proposed for the classical implicit functions theorem, involving implicit parametrizations in finite dimensional spaces. While it is intuitive that implicit parametrizations offer, in general, a more advantageous representation of implicitly defined manifolds, the representation is even global in some important cases. For instance, in dimension two, in the general setting of the Poincaré-Bendixson theorem [4, 14], the implicit parametrization that we construct, is always global [18]. In dimension three, we quote the example of the torus, from [10]. In fact, this is an important question for the applications: when have the implicit parametrizations a global character, also in dimension three?

This new representation of manifolds (of arbitrary dimension and codimension) was intended for applications in geometric optimization problems and we quote $[18$, $7,21,22]$ for recent results in this respect. It turns out that it is also useful in mathematical programming and in optimal control as shown in [23, 20, 25].

In this paper, we briefly review such results and their possible extensions. The Section 2 is devoted to the implicit parametrization question. In Section 3, applications in nonlinear programming and optimal control are briefly discussed. The last section 
includes some relevant properties obtained in shape optimization and their possible generalizations.

\section{Implicit parametrizations}

We consider a system of $l<d$ equalities defined in some bounded open set $\Omega \subset R^{d}$, with $l, d$ natural numbers:

$$
F_{1}(\bar{x})=F_{2}(\bar{x})=\ldots=F_{l}(\bar{x})=0, \bar{x} \in \Omega .
$$

Above we assume that $F_{1}, F_{2}, \ldots, F_{l} \in C^{1}(\bar{\Omega})$ and there is $\bar{x}_{0} \in \Omega$ such that (2.1) is satisfied and

$$
\frac{D\left(F_{1}, F_{2}, \ldots, F_{l}\right)}{D\left(x_{1}, x_{2}, \ldots, x_{l}\right)} \neq 0 \text { in } \bar{x}_{0}=\left(x_{1}^{0}, x_{2}^{0}, \ldots, x_{d}^{0}\right) .
$$

Notice that (2.2) remains valid on a neighbourhood $V$ of $\bar{x}_{0}, V \subset \Omega$. In $V$, we define the linear algebraic system

$$
v(\bar{x}) \cdot \nabla F_{j}(\bar{x})=0, j=\overline{1, l},
$$

where the unknown vector $v(\bar{x}) \in R^{d}$.

It is known that (2.1), under condition (2.2), defines a $d-l$ dimensional manifold contained in $\Omega$ and $\nabla F_{1}(\bar{x}), \nabla F_{2}(\bar{x}), \ldots, \nabla F_{l}(\bar{x})$ are a basis in the normal space at $\bar{x}$, to this manifold.

Therefore, any solution to (2.3) is a vector in the tangent space to this manifold and we fix $v_{1}, v_{2}, \ldots, v_{d-l}$ as continuous (in $V$ ) independent solutions of (2.3) that is a basis in the tangent space to the manifold. The choice of $v_{1}, v_{2}, \ldots, v_{d-l}$ is not unique [19].

We associate to them a system of nonlinear partial derivatives of order one:

$$
\begin{gathered}
\frac{\partial y_{1}\left(t_{1}\right)}{\partial t_{1}}=v_{1}\left(y_{1}\left(t_{1}\right)\right), t_{1} \in I_{1} \subset R, y_{1}(0)=\bar{x}_{0} ; \\
\frac{\partial y_{2}\left(t_{1}, t_{2}\right)}{\partial t_{2}}=v\left(y_{2}\left(t_{1}, t_{2}\right)\right), t_{2} \in I_{2}\left(t_{1}\right) \subset R, y_{2}\left(t_{1}, 0\right)=y_{1}\left(t_{1}\right) ; \\
\ldots \\
\ldots \\
\frac{\partial y_{d-l}\left(t_{1}, t_{2}, \ldots, t_{d-l}\right)}{\partial t_{d-l}}=v_{d-l}\left(y_{d-l}\left(t_{1}, t_{2}, \ldots, t_{d-l}\right)\right), \\
t_{d-l} \in I_{d-l}\left(t_{1}, t_{2}, \ldots, t_{d-l}\right), \\
y_{d-l}\left(t_{1}, t_{2}, \ldots, t_{d-l-1}, 0\right)=y_{d-l-1}\left(t_{1}, t_{2}, \ldots, t_{d-l-1}\right) .
\end{gathered}
$$

The system (2.4) - (2.6) has an iterated character.

Each equation has one supplementary independent variable and the initial condition corresponding to it is given by the solution of the previous equation (by $\bar{x}_{0}$ in the first one). Moreover, each equation includes just one derivative, therefore (2.4) (2.6) is in fact a system of $d-l$ ordinary differential subsystems, each of dimension $d$. By $I_{1}, I_{2}\left(t_{1}\right), \ldots, I_{d-l}\left(t_{1}, t_{2}, \ldots, t_{d-l}\right)$ we denote the corresponding existence intervals, 
around the origin. The existence is ensured by the Peano theorem, due to the continuity of $v_{1}, v_{2}, \ldots, v_{d-l}$. The independent variables not involved in derivation, play the role of parameters and they enter just via the initial condition. The numerical solution via Matlab is standard and easy.

Furthermore, each of the system $(2.4),(2.5), \ldots,(2.6)$ solves an inverse problem: given $F_{1}, F_{2}, \ldots, F_{l} \in C^{1}(\bar{\Omega})$ with conditions $(2.1),(2.2)$, the mentioned systems are constructed in such a way that $F_{1}, F_{2}, \ldots, F_{l}$ are prime integrals for any of them (see [19]).

Theorem 2.1. For every $k=\overline{1, l}, j=\overline{1, d-l}$, we have

$$
F_{k}\left(y_{j}\left(t_{1}, t_{2}, \ldots, t_{j}\right)\right)=0 \text {, }
$$

for any $\left(t_{1}, t_{2}, \ldots, t_{j}\right) \in I_{1} \times I_{2}\left(t_{1}\right) \times \ldots \times I_{j}\left(t_{1}, t_{2}, \ldots, t_{j-1}\right)$.

Due to the conservation property in (2.7) and to the examples in [17], [10], we call such systems to be of Hamiltonian type. They have unexpected properties.

Theorem 2.2. Under condition (2.2), each system has the uniqueness property in $V$, the intervals $I_{2}\left(t_{1}\right), \ldots, I_{d-l}\left(t_{1}, t_{2}, \ldots, t_{d-l-1}\right)$ may be chosen independently of the parameters and the unique solutions of $(2.4),(2.5), \ldots,(2.6)$ are of class $C^{1}$ in each of their arguments and

$$
\frac{\partial y_{d-l}}{\partial t_{k}}\left(t_{1}, t_{2}, \ldots, t_{d-l}\right)=v_{k}\left(y_{d-l}\left(t_{1}, \ldots, t_{d-l}\right)\right), k=\overline{1, d-l} .
$$

Relation (2.8) is immediately extended to $y_{1}, \ldots, y_{d-l-1}$ due to the initial conditions in $(2.4),(2.5), \ldots,(2.6)$.

The most important property obtained via $(2.4),(2.5), \ldots,(2.6)$ is the following.

Theorem 2.3. Under the above assumptions, the mapping

$$
y_{d-l}: I_{1} \times I_{2} \times \ldots \times I_{d-l} \rightarrow R^{d}
$$

is regular and one-to-one on its image.

That is, $y_{d-l}$ gives a parametrization of the manifold (2.1) around $\bar{x}$. In [19], it is also shown that the classical implicit functions theorem may be obtained as well as a special case of the above constructive approach. However, as we have already argued, parametrizations offer a more complete description of the manifold.

We also recall that the classical hypothesis (2.2) may be dropped and a generalized solution of the system (2.1) may be introduced and studied according to [17], [19].

In dimension two, the iterated system $(2.4),(2.5), \ldots,(2.6)$ becomes the simplest Hamiltonian system associated to some $g \in C^{1}(\bar{\Omega})$, a new notation of $F_{1}$, such that $g\left(\bar{x}_{0}\right)=0, \nabla g\left(\bar{x}_{0}\right) \neq 0, \bar{x}_{0} \in \Omega \subset R^{2}$, which correspond to the conditions $(2.1),(2.2)$ :

$$
\begin{gathered}
x_{1}^{\prime}(t)=-\frac{\partial g}{\partial x_{2}}\left(x_{1}(t), x_{2}\left(t_{1}\right)\right), t \in I, \\
x_{2}^{\prime}(t)=\frac{\partial g}{\partial x_{1}}\left(x_{1}(t), x_{2}\left(t_{1}\right)\right), t \in I, \\
\left(x_{1}(0), x_{2}(0)\right)=\bar{x}_{0} .
\end{gathered}
$$


Obviously, the Theorem 2.1, Theorem 2.2, Theorem 2.3 remain valid for the system (2.9), (2.10), including relations (2.7), (2.8). We introduce now the hypothesis

$$
\left|\nabla g\left(x_{1}, x_{2}\right)\right|>0 \text { on } G=\left\{\left(x_{1}, x_{2}\right) \in \Omega ; g\left(x_{1}, x_{2}\right)=0\right\},
$$

which is a reformulation of the hypothesis in the Poincaré - Bendixson theorem [4], [14], for (2.9), (2.10).

For convenience, we also assume that

$$
g\left(x_{1}, x_{2}\right)>0 \text { on } \partial \Omega \text {. }
$$

Theorem 2.4. Under conditions (2.11),(2.12), $G$ is a finite union of disjoint closed curves, without self intersections and not intersecting $\partial \Omega$, parametrized by the solution of $(2.9),(2.10)$, when some initial condition $\bar{x}_{0}$ is chosen on each of its components.

This result was proved in [18] and gives the global existence and the periodicity of the solution for the Hamiltonian system (2.9), (2.10). It has an important role in the analysis of shape optimization problems in dimension two, which is a case of interest [18], [7].

Remark 2.5. The question of the extension of Thm. 2.4 to dimension three or higher, is open, [10]. This is mainly due to the fact that the Poincare-Bendixson theorem is valid just in dimension two. The extension (of interest in the setting of shape optimization problems) refers to the iterated Hamiltonian systems (2.4), (2.5), ... (2.6) and consists of finding reasonable sufficient conditions ensuring that the obtained manifold is closed and the representation via $(2.4),(2.5), \ldots,(2.6)$ is global.

\section{Optimization and optimal control}

We discuss here the general constrained nonlinear programming problem in $R^{d}$ :

$$
\operatorname{Min}\left\{h\left(x_{1}, \ldots, x_{d}\right)\right\}
$$

subject to (2.1) and to inequality constraints

$$
G_{j}\left(x_{1}, \ldots, x_{d}\right) \leq 0, j=\overline{1, m},
$$

where $h, F_{i}, i=\overline{1, l}, G_{j}, j=\overline{1, m}$ are in $C^{1}\left(R^{d}\right)$ and the classical Mangasarian Fromowitz assumption, see [1], is valid.

That is (2.2) is assumed and there is $e \in R^{d}$ such that

$$
\nabla F_{i}\left(\bar{x}_{0}\right) e=0, i=\overline{1, l}, \nabla G_{j}\left(\bar{x}_{0}\right) e<0, j \in I\left(\bar{x}_{0}\right) .
$$

Here, $I\left(\bar{x}_{0}\right)$ is the set of indices $j=\overline{1, m}$ of the active inequality constraints at $\bar{x}_{0}$. By using Thm. 2.3 (the special case of implicit functions, when the parametrization $y_{d-l}$ has the last $d-l$ components given by the coordinates in $R^{d}$, see [19]) we obtain the reduced optimization problem that involves just inequality constraints:

$$
\operatorname{Min}\left\{h\left(y_{d-l}^{1}, y_{d-l}^{2}, \ldots, y_{d-l}^{l}, t_{1}+x_{l+1}^{0}, \ldots, t_{d-l}+x_{d}^{0}\right)\right\}
$$

subject to

$$
G_{j}\left(y_{d-l}^{1}, y_{d-l}^{2}, \ldots, y_{d-l}^{l}, t_{1}+x_{l+1}^{0}, \ldots, t_{d-l}+x_{d}^{0}\right) \leq 0, j=\overline{1, m},
$$


where $\left(t_{1}, \ldots, t_{d-l}\right)$ is in a neighbourhood of the origin in $R^{d-l}$.

It turns out (see [23]) that the reduced problem (3.4), (3.5) also satisfies the Mangasarian - Fromowitz condition (3.3) (adapted to this setting) in the origin of $R^{d-l}$. Using derivation formulas as in (2.8), we get

Theorem 3.1. Let $\bar{x}_{0}$ be a local solution of the problem (3.1), (2.1), (3.2). Then, there are $\beta_{j} \geq 0, j=\overline{1, m}$ such that

$$
\begin{gathered}
0=\nabla h\left(\bar{x}_{0}\right) \cdot v_{s}\left(\bar{x}_{0}\right)+\sum_{j=1}^{m} \beta_{j} \nabla G_{j}\left(\bar{x}_{0}\right) \cdot v_{s}\left(\bar{x}_{0}\right), s=\overline{1, d-l}, \\
0=\beta_{j} G_{j}\left(\bar{x}_{0}\right), j=\overline{1, m} .
\end{gathered}
$$

This is a simplified version of the KKT optimality conditions since the multipliers associated to (2.1) are eliminated. If we consider just the optimization problem with equality constraints (3.1), (2.1), then (finally) we obtain the optimality conditions in Fermat form by taking $\beta_{j}=0, j=\overline{1, m}$, in Thm. 3.1.

Notice that the first term in (3.6) is the tangential derivative of the cost in $\bar{x}_{0}$ (the components of $\nabla h\left(\bar{x}_{0}\right)$ from the tangent plane to the manifold of equality constraints). This allows to formulate an algorithm of gradient type with projection for the problem $(3.1),(2.1)$. The novelty here is that the projection can be effectively computed as $y_{d-l}$ in each step of this algorithm.

Details in [23], and we underline that the question of the computation of the projection is this main drawback for this type of numerical methods, Ciarlet [2]. Moreover, in the general case of the problem (3.1), (2.1), (3.2) we notice that the inequality constraints that are not active at $\bar{x}_{0}$ define a neighbourhood of $\bar{x}_{0}$. One can reformulate equivalently the problem on this neighbourhood and involving just the equality constraints (2.1) and the equality constraints obtained from the active inequality constraints. Therefore, the conditions (3.6), (3.7) reduce to (3.6) with $\beta_{j}=$ $1, j \in I\left(\bar{x}_{0}\right)$ and $\beta_{j}=0$ otherwise and $\left\{v_{s}\right\}$ restricted to include just the basis of the tangent space to the manifold defined by all these equality constraints (assumed independent in $\bar{x}_{0}$ ).

Using the maximal (in space) description offered by Thm. 2.3, of the manifold defined by (2.1), we introduce a class of algorithms of "global type". Namely, they search for solution in a maximal admissible neighbourhood of $\bar{x}_{0}$ (which is just an admissible point here) and may find all the solutions from this admissible set.

The basic observation is that the solution of $(2.4),(2.5), \ldots,(2.6)$ exists (roughly speaking), up to the "moment" of meeting a critical point. And the computation of $y_{d-l}$ on this maximal existence interval, for a finer and finer discretization, provides a dense set of points in the manifold defined by (2.1). We denote it by $A_{n}$, in the $n$-th discretization step. The constraints (3.2) are to be just checked on this points in $A_{n}$. We may impose even supplementary abstract constraints in the problem (3.1), (2.1), (3.2), of the form:

$$
\bar{x} \in D, D \subset R^{d} \text { closed subset. }
$$

We denote by $C_{n}$ the discrete admissible set of points, in step $n$, obtained after checking the points in $A_{n}$ for (3.2) and (3.8). The algorithm is as follows: 
Algorithm 3.2

1) choose $n=1$, the discretization step $\frac{1}{n}$ in (3.4) - (3.6) and the solution intervals $I_{1}^{n}, \ldots, I_{d-l}^{n}$, the small parameter $\delta$.

2) compute $A_{n}$ and $C_{n}$.

3) find in $C_{n}$, the minimum of $h(\cdot)$, by direct computation, denoted by $x_{n}$.

4) test $\left|h\left(x_{n}\right)-h\left(x_{n-1}\right)\right|<\delta$.

5) If YES, then STOP; if NO, then GO TO step 1).

In this setting, it is enough to assume $h$ and $G_{j}, j=\overline{1, m}$ to be in $C\left(R^{d}\right)$, $F_{i}, i=\overline{1, l}$ satisfy $(2.2)$ and $D$ has nonvoid interior. By density, we get

Theorem 3.2. The algorithm is convergent for $n \rightarrow \infty$.

The admissible set for the problem (3.1), (2.1) may have several connected components, see [23].

Then, it is necessary to know an initial point $\bar{x}_{0}$ on each component, for the algorithm to work. If hypothesis (2.2) is not fulfilled, we suggest to work with generalized solutions of $(2.4),(2.5), \ldots,(2.6)$. This subject is not yet investigated in the literature.

Finally, we mention the recent paper [16], that proposes an alternative approach in similar situation. We have reworked in [23] their main numerical example (in $R^{6}$ ) by employing implicit parametrizations and starting from the solutions they found. Our investigation allows a very consistent decrease of the optimal value for the performance index. The Algorithm 3.2 easily allows to extend the search region, simply by increasing the computations intervals for $(2.4),(2.5), \ldots,(2.6)$. Some high dimensional applications are also reported in [9].

Similar ideas work for constrained optimal control problems.

Here, we briefly discuss the difficult case of mixed equality constraints, following [23]. The problem, of Mayer type, is the following:

$$
\begin{gathered}
\operatorname{Min}\{l(x(0), x(1))\}, \\
x^{\prime}(t)=f(t, x(t), u(t)), t \in[0,1], \\
h(x(t), u(t))=0, t \in[0.1],
\end{gathered}
$$

and it is inspired from the recent works [3], [12], where the maximum principle is discussed.

Here $l(\cdot, \cdot), f(t, \cdot, \cdot), h(\cdot, \cdot)$ are defined in finite dimensional spaces $X \times X, X \times U$ of appropriate dimension and (3.10) is assumed to be uniquely and globally solvable, as it is standard in optimal control theory. Later, we shall add to it initial conditions. We require $l$ continuous, $f$ continuous and locally Lipschitzian in $(x, u), h$ of class $C^{1}$, with locally Lipschitzian gradient for each component, such that there is the vector $\left(x^{0}, u^{0}\right)$ in $X \times U$ satisfying

$$
h\left(x^{0}, u^{0}\right)=0, \nabla h\left(x^{0}, u^{0}\right) \text { of maximal rank. }
$$

The finite dimensional nonlinear algebraic system $h(x, u)=0$, under condition (3.12), defines a manifold $M \subset X \times U$ that can be parametrized and discretized 
via the system $(2.4),(2.5), \ldots,(2.6)$. Moreover, the relations (3.10), (3.11) can be interpreted as a DAE system and we differentiate (3.11) and replace it by:

$$
\nabla_{x} h(x(t), u(t) f(t, x(t)), u(t))+\nabla_{u} h(x(t), u(t)) u^{\prime}(t)=0 .
$$

The important remark is that the manifold $M$ provides consistent initial conditions for (3.10), (3.13).

Theorem 3.3. Any trajectory of (3.10), (3.13) starting from a point in $M$, remains in $M$.

This gives a characterization of the admissible global trajectories for the constrained control problem (3.9) - (3.11). Consequently, it can be shown that the discretization of $M$ provided by $(2.4),(2.5), \ldots,(2.6)$, generates a dense family of admissible trajectories and an algorithm of global type, similar to Algorithm 3.2, can be formulated and its convergence remains valid [23].

Some academic examples can be found in [23] as well.

\section{Shape optimization}

Shape optimization problems have a similar structure with optimal control problems, for instance:

$$
\begin{gathered}
\operatorname{Min}_{\Omega} \int_{\Omega} j(x, y(x)) d x, \\
-\Delta y=f \text { in } \Omega, \\
y=0 \text { on } \partial \Omega .
\end{gathered}
$$

Here $f \in L^{2}(D)$ and $j(\cdot, \cdot)$ is a Caratheodory mapping, the admissible domains satisfy $\Omega \subset D, D$ a given domain bounded in $R^{d}$.

Other elliptic operators (or even evolution operators, [24]), other boundary conditions or cost functionals (defined on $\partial \Omega$, or on some given subset $E \subset \Omega$, for any $\Omega$ admissible, or depending as well on $\nabla y(x)$, etc.), more constraints (for instance, on the state $y$ ) may be considered in (4.1) - (4.3).

An important choice is the admissible family of geometries in $R^{d}$, denoted by $\mathcal{O}$. In one of the first approaches in shape optimization, due to Murat and Simon [6], the family $\mathcal{O}$ is given as the image of some fixed domain $B \subset R^{d}$ (for instance, a ball) via a family $\mathcal{F}$ of mapping $T: B \rightarrow R^{d}$, of class $C^{2}$, one-to-one on their image $T(B)$ and $T^{-1}$ of class $C^{2}$ as well.

Then, (4.1) - (4.3) may be transported on $B$ and the transformation $T \in \mathcal{F}$ will enter, together with its derivatives, in the coefficients of the transformed elliptic operator in $B$. The geometric optimization problem (4.1) - (4.3) is then equivalent with a control by the coefficients problem, if $\mathcal{O}$ is defined as above. The drawback of this approach is that all the admissible domains $\Omega \in \mathcal{O}$ have to be simply connected (when $B$ is a ball), that is this family $\mathcal{O}$ is not general enough.

A similar discussion may be pursued in the case of the speed method of Zolesio, [26]. See [13], [15], [8] for information in this respect. 
A more far reaching point of view is to assume that the admissible domains $\Omega \in \mathcal{O}$ are given via an implicit representation, using a family of functions $\Phi \in \mathcal{F}$ :

$$
\Omega=\Omega_{\Phi}=\left\{x \in R^{d} ; \Phi(x)<0, \Phi \in \mathcal{F}\right\},
$$

where $\mathcal{F}$ is now a subset in $C(\bar{D})$ with $D \subset R^{d}$ some given bounded domain. Obviously, relation (4.4) defines an open set and supplementary information should be given in order to select some connected component of interest, not necessarily simply connected. In this way, both topological and boundary variations may be considered in the problem (4.1) - (4.3).

This point of view was introduced by Osher and Sethian [11] in the setting of free boundary problems and the treatment is based on the Hamilton - Jacobi equation.

In shape optimization, implicit representation of domains were considered independently, already in [5]. Recently, it was shown that iterated Hamiltonian type systems, as discussed in $\S 2$, play a fundamental role in this setting [18]. Such ordinary differential systems are much easier to handle as Hamilton - Jacobi equations and Thm. 2.4 is the key result in dimension two, which is a case of interest in shape optimization.

A frequently met supplementary constraint on the admissible $\Omega \in \mathcal{O}$ is $E \subset \Omega$, where $E \subset D$ is another given open subset.

This geometric condition, under definition (4.4), is expressed as $\Phi(x)<0$ in $E$, a very simple algebraic condition. Notice that it also selects the connected component of $\Omega_{\Phi}$, that is the domain of interest in the optimization problem. Similarly, one may ask that, for a given point $x_{0} \in D$, we have $x_{0} \in \partial \Omega_{\Phi}$ (or for some given submanifold $\Gamma \subset D$, we have $\left.\Gamma \subset \partial \Omega_{\Phi}\right)$. This is expressed algebraically as $\Phi\left(x_{0}\right)=0$ (or $\Phi(x)=0$ on $\Gamma$ ) and again selects in (4.4) the connected component of interest of $\Omega_{\Phi}$.

We underline that, if we assume just $\mathcal{F} \subset C(\bar{D})$, then $\partial \Omega_{\Phi}$ may have positive measure. Under condition (2.11), this cannot happen and the above examples are clearly defined, while the facility to translate geometric constraints in simple algebraic conditions is remarkable.

It turns out that the geometric optimization problem (4.1) - (4.3) is equivalent with a state constraint optimal control problem in $D$, for $\mathcal{O}$ given by (4.4).

Theorem 4.1. Assume (2.11), (2.12) and let $\Omega_{\Phi}$ be defined by (4.4). For any $\Phi \in \mathcal{F}$, there is $u_{\Phi} \in L^{2}(D)$ (not unique) such that the solution of

$$
\begin{gathered}
-\Delta y=f+H(\Phi) u_{\Phi} \text { in } D, \\
y=0 \text { on } \partial D,
\end{gathered}
$$

coincides in $\Omega_{\Phi}$ with the solution of (4.2),(4.3) and satisfies the constraint

$$
\int_{\partial \Omega_{\Phi}}|y(\sigma)|^{2} d \sigma=0 .
$$

The cost (4.1) corresponding to $\Omega_{\Phi}$ is identical with the cost associated to $\left[y_{\phi}, \Phi, u_{\Phi}\right]$ given in $(4.5),(4.6)$. 
Here, $H(\cdot): R \rightarrow R$ is the Heaviside function.

The condition (4.7) may be expressed in the form (independently of the geome$\operatorname{try})$ :

$$
\int_{0}^{T_{\Phi}}\left|y\left(x_{1}(t), x_{2}(t)\right)\right|^{2} \sqrt{x_{1}^{\prime}(t)^{2}+x_{2}^{\prime}(t)^{2}} d t=0,
$$

where $\left(x_{1}(t), x_{2}(t)\right)$ solves $(2.9),(2.10)$ on the period $\left[0, T_{\Phi}\right]$ and $\bar{x}_{0}$ is some fixed given point on $\partial \Omega_{\Phi}$. Moreover, the cost functional (4.1) may be also rewritten in an "independent of the geometry" form:

$$
\int_{D}(1-H(\Phi)) j(x, y(x)) d x
$$

and, in fact, $H(\Phi)$ is the characteristic function of $D \backslash \Omega_{\Phi}$, under hypotheses (2.11), (2.12).

Consequently, the optimal control problem (4.5), (4.6), (4.8), (4.9) (with controls $\left.\Phi \in \mathcal{F}, u \in L^{2}(D)\right)$ is independent of the geometry and is equivalent with the shape optimization problem $(4.1)-(4.3)$, on $\mathcal{O}$ defined by $\mathcal{F}$ via $(4.4)$.

A standard procedure in state constrained control problem is the penalization of the constraint in the cost $(\varepsilon>0)$ :

$$
\int_{D}(1-H(\Phi)) j(x, y(x)) d x+\frac{1}{\varepsilon} \int_{0}^{T_{\Phi}}\left|y\left(x_{1}(t), x_{2}(t)\right)\right|^{2} \sqrt{x_{1}^{\prime}(t)^{2}+x_{2}^{\prime}(t)^{2}} d t .
$$

General approximation properties of the problem (4.5), (4.6), (4.10) with respect to the constrained problem $(4.5),(4.6),(4.8),(4.9)$ or to the original shape optimization problem $(4.1),(4.2),(4.3)$, are discussed in [18]. We indicate here just one property, when the cost integrand in (4.1) depends as well on $\nabla y, j(x, y(x), \nabla y(x))$.

Theorem 4.2. Assume that $j(\cdot, \cdot, \cdot)$ is Caratheodory on $D \times R \times R^{2}$ and satisfies the coercivity assumption

$$
j(x, y, v) \geq \alpha_{1}|v|^{2}+\beta_{1}|y|^{2}+\gamma, \alpha_{1}>0, \beta_{1}>0, \gamma \in R
$$

and $j(x, y, \cdot)$ is convex. Then, if $\left[y_{n}^{\varepsilon}, \Phi_{n}^{\varepsilon}, u_{n}^{\varepsilon}\right]$ denote a minimizing sequence in the penalized problem (4.5), (4.6), (4.10) and $y^{*}, \Omega^{*}$ are cluster points of the sequence $\left[y_{n}^{\varepsilon}, \Omega_{\Phi_{n}^{\varepsilon}}\right]$ in the weak topology of $L^{2}(D)$, respectively in the Hausdorff - Pompeiu complementary topology, then $\left[y^{*}, \Omega^{*}\right]$ is an optimal pair for the problem (4.1) - (4.3).

The technique employed in [18] includes as well a modification of $\left\{y_{n}^{\varepsilon}\right\}$ outside $\Omega_{\Phi_{n}^{\varepsilon}}$. In the paper [7], a differentiable variant of this approach is studied. The implicit parametrization theorem gives a global representation of the boundary and allows to compute integrals as in $(4.7),(4.8),(4.10)$. It also allows to discuss boundary observation problems [22].

Remark 4.3. One question of interest, in this context, is to obtain efficient gradient algorithms, in general shape optimization problems. Certain results of this type are reported in [7], for Dirichlet boundary conditions. Another question is related to the 
possibility to use just one control in the "extension" (4.5), (4.6) of the state system, while preserving all the other properties.

\section{References}

[1] Bonnans, J.F., Shapiro, A., Perturbation Analysis of Optimization Problems, Springer Verlag, New York, 2000.

[2] Ciarlet, P.G., Introduction to Numerical Linear Algebra and Optimization, Cambridge Univ. Press, Cambridge, 1989.

[3] Clarke, F., de Pinho, M.R., Optimal control problems with mixed constraints, SIAM J. Control Optim., 48(2011), 4500-4524.

[4] Hirsch, M.W., Smale, S., Devaney, R.L., Differential Equations, Dynamical Systems and Introduction to Chaos, 3rd ed., Elsevier, Academic Press, New York, 2013.

[5] Mäkinen, R., Neittaanmäki, P., Tiba, D., On a fixed domain approach for a shape optimization problem, in: Computational and applied Mathematics II: Differential equations (W.F. Ames, P.J. van Houwen, eds.), North Holland, Amsterdam, 1992, 317-326.

[6] Murat, F., Simon, J., Etude de problèmes d'optimal design, in: Optimization techniques. Modeling and optimization in the service of man. Part 2 (J. Céa, ed.), LNCS41, Springer Verlag, Berlin, 1976, 54-62.

[7] Murea, C., Tiba, D., Topological optimization via cost penalization, TMNA, 54(2019), no. 2B, 1023-1050.

[8] Neittaanmäki, P., Sprekels, J., Tiba, D., Optimization of Elliptic Systems. Theory and Applications, Springer Verlag, New York, 2006.

[9] Nicolai, M.R., High dimensional applications of implicit parametrizations in nonlinear programming, Ann. Acad. Rom. Sci. Ser. Math. Appl., 8(2016), 44-55.

[10] Nicolai, M.R., Tiba, D., Implicit functions and parametrization in dimension three: generalized solutions, Discrete Contin. Dyn. Syst., 35(2015), 2701-2710.

[11] Osher, S., Sethian, J.A., Fronts propagating with curvature-dependent speed; algorithms based on Hamiltonian-Jacobi formulation, Journal of Computational Physics, 79(1988), no. $1,12-49$.

[12] de Pinho, M.R., On necessary conditions for implicit control systems, Pure Appl. Funct. Anal., 1(2016), 185-196.

[13] Pironneau, O., Optimal Shape Design for Elliptic Systems, Springer Verlag, Berlin, 1984.

[14] Pontryagin, L.S., Equation Differentielles Ordinaires, MIR, Moscow, 1968.

[15] Sokolovski, J., Zolesio, J.-P., Introduction to Shape Optimization. Shape Sensitivity Analysis, Springer Verlag, Berlin, 1992.

[16] Stuber, M.D., Scott, J.K., Bartar, P.I., Convex and concave relaxations of implicit functions, Optimization Methods and Software, 30(2015), 424-460.

[17] Tiba, D., The implicit functions theorem and implicit parametrizations, Ann. Acad. Rom. Sci. Ser. Math. Appl., 5(2013), 193-208.

[18] Tiba, D., A penalization approach in shape optimization, Atti. Acad. Pelorit. Pericol. Cl. Sci. Fis. Mat. Nat., 16(2018), no. 1, A8.

[19] Tiba, D., Iterated Hamiltonian type systems and applications, JDE, 264(2018), 54655479 . 
[20] Tiba, D., Some remarks on state constraints and mixed constraints, Ann. Acad. Rom. Sci. Ser. Math. Appl., 10(2018), 25-40.

[21] Tiba, D., Neumann boundary conditions in shape optimization, PAFA, 3(2018), 241-253.

[22] Tiba, D., Boundary observation in shape optimization, in: New Trends in Differential Equations, Control Theory and Optimization (V. Barbu, C. Lefter, I. Vrabie, Eds.), World Scientific, Singapore, 2016, 301-315.

[23] Tiba, D., Implicit parametrizations and applications in optimization and control, MCRF, 10(2020), 455-470.

[24] Tiba, D., Yamamoto, M., A parabolic shape optimization problem, Ann. Acad. Rom. Sci. Ser. Math. Appl., 12(2020), 312-328.

[25] Tiba, D., Zălinescu, C., On the necessity of some constraint qualification conditions in convex programming, J. Convex Anal., 11(2004), 95-110.

[26] Zolesio, J.-P., Identification de Domaines par Deformation, Thèse, Univ. of Nice, France, 1979.

Dan Tiba

"Simion Stoilow" Institute of Mathematics of the Romanian Academy

and

Academy of Romanian Scientists,

Bucureşti, Romania

e-mail: dan.tiba@imar.ro 